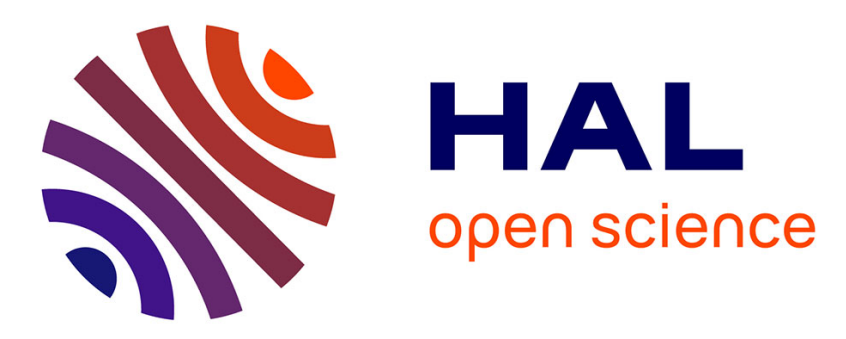

\title{
Surface Diffusion of Cr Adatoms on Au(111) by Quantum Tunneling
}

Philippe Ohresser, Hervé Bulou, S.S. Dhesi, C. Boeglin, Bence Lazarovits, Emilie Gaudry, Idrissou Chado, J. Faeber, Fabrice Scheurer

\section{- To cite this version:}

Philippe Ohresser, Hervé Bulou, S.S. Dhesi, C. Boeglin, Bence Lazarovits, et al.. Surface Diffusion of $\mathrm{Cr}$ Adatoms on $\mathrm{Au}(111)$ by Quantum Tunneling. Physical Review Letters, 2005, 95, pp.195901. 10.1103/PhysRevLett.95.195901 . hal-00978036

\section{HAL Id: hal-00978036 https://hal.science/hal-00978036}

Submitted on 11 Apr 2014

HAL is a multi-disciplinary open access archive for the deposit and dissemination of scientific research documents, whether they are published or not. The documents may come from teaching and research institutions in France or abroad, or from public or private research centers.
L'archive ouverte pluridisciplinaire HAL, est destinée au dépôt et à la diffusion de documents scientifiques de niveau recherche, publiés ou non, émanant des établissements d'enseignement et de recherche français ou étrangers, des laboratoires publics ou privés. 


\title{
Surface Diffusion of Cr Adatoms on Au(111) by Quantum Tunneling
}

\author{
P. Ohresser, ${ }^{1, *}$ H. Bulou, ${ }^{2}$ S. S. Dhesi, ${ }^{3, \dagger}$ C. Boeglin, ${ }^{2}$ B. Lazarovits, ${ }^{4}$ E. Gaudry, ${ }^{5}$ I. Chado, ${ }^{6}$ J. Faerber, ${ }^{2}$ and F. Scheurer ${ }^{2}$ \\ ${ }^{1}$ Laboratoire pour l'Utilisation du Rayonnement Electromagnétique, UMR 130 CNRS-Université Paris Sud, 91898 Orsay (France) \\ ${ }^{2}$ Institut de Physique et Chimie des Matériaux de Strasbourg, UMR 7504 CNRS-Université Louis Pasteur, \\ 23 rue du Loess, 67034 Strasbourg (France) \\ ${ }^{3}$ European Synchrotron Radiation Facility, BP 200, F-38043 Grenoble, France \\ ${ }^{4}$ Center for Computational Materials Science, Technical University Vienna, Gumpendorferstrasse 1a, 1060 Wien (Austria) \\ ${ }^{5}$ Laboratoire de Minéralogie et Cristallographie de Paris, 4 place de Jussieu, 75252 Paris (France) \\ ${ }^{6}$ Physikalisches Institut, Universität Würzburg, Am Hubland, 97074 Würzburg, (Germany)
}

(Received 1 August 2005; published 1 November 2005)

\begin{abstract}
The low-temperature surface diffusion of isolated $\mathrm{Cr}$ adatoms on $\mathrm{Au}(111)$ has been determined using nonperturbing $\mathrm{x}$ rays. Changes in the $\mathrm{x}$-ray magnetic circular dichroism spectral line shape together with Monte Carlo calculations demonstrate that adatom nucleation proceeds via quantum tunneling diffusion rather than over-barrier hopping for temperatures $<40 \mathrm{~K}$. The jump rates are shown to be as much as 35 orders of magnitude higher than that expected for thermal over-barrier hopping at $10 \mathrm{~K}$.
\end{abstract}

DOI: 10.1103/PhysRevLett.95.195901

Quantum tunneling is one of the most intriguing properties of matter and fundamental in numerous phenomena in physics and chemistry. Among the most outstanding advances allowed by quantum tunneling, one finds atomic imaging [1] that led to atomic manipulation and the discovery of quantum mirages [2], and more recently, cascading surface molecules used to operate elementary nanoscale computers [3]. Classically, a particle is trapped unless it gains enough energy to overcome the surrounding energy barrier, but quantum mechanically a particle can tunnel through the barrier provided that its de Broglie wavelength [4] is large enough. Since the de Broglie wavelength decreases with particle mass and temperature, surface quantum tunneling has so far been observed only for light particles. For instance, hydrogen and its isotopes, deposited on metal surfaces, exhibit a clear transition from thermal over-barrier hopping to quantum tunneling diffusion [5-9]. In principle, quantum tunneling diffusion should be observable for much heavier surface adatoms, but this requires a low surface potential corrugation, a highly sensitive nonperturbing probe, and a surface evolving at a detectable rate. Isolated surface adatoms, which nucleate to form stable clusters of several adatoms, provide an ideal system with which to study quantum tunneling.

Upon clustering, charge transfer and hybridization leads to distinct changes in electronic structure which can be determined using x-ray absorption spectroscopy (XAS). Unfortunately, subtle changes in spectroscopic measurements are often difficult to detect. However, x-ray magnetic circular dichroism (XMCD), which measures the difference between two XAS spectra recorded with oppositely polarized $\mathrm{x}$ rays, can detect unitary changes in surface adatom coordination $[10,11]$ making it ideal to study surface diffusion. The reason for this is that XMCD involves selection rule governed transitions from core levels to unoccupied states just above the Fermi level. These unoccupied states are highly sensitive to the local atomic
PACS numbers: 61.50.Ah, 66.10.Cb, 68.35.Fx, 68.43.Jk

coordination [12]. Whereas single $3 d$ adatoms exhibit sharp multiplet peaks in the $L_{2,3}$ XMCD spectrum [10], coordinated adatoms exhibit much broader band structure features. Compared to diffusion coefficients measured using field ion microscopy or scanning tunneling microscopy [13], the benefit of XMCD is the large spatial averaging $\left(10^{5} \mu \mathrm{m}^{2}\right)$ and the nonperturbative $\mathrm{x}$-ray approach.

An $\mathrm{Au}(111)$ surface combines a low potential barrier height $[14,15]$ and a close packed array of adsorption sites which should make quantum tunneling diffusion rates comparable to thermal diffusion rates at low temperatures. A collection of initially isolated adatoms, obtained by depositing minute amounts of $\mathrm{Cr}$ at $10 \mathrm{~K}$, has a particular XMCD spectral line shape which can be changed by adatom aggregation via deposition of additional material $[10,11]$. On the other hand, the exact same changes in the $\mathrm{XMCD}$ line shape can be realized by measuring the system again after a few thousand seconds depending on the surface temperature. If the surface temperature is raised then the evolution of the line shape is faster, but exactly the same XMCD spectra are obtained.

In this Letter, we demonstrate surface quantum tunneling diffusion of $\mathrm{Cr}$ adatoms on an $\mathrm{Au}(111)$ surface using the time evolution of XMCD spectra measured at the $\mathrm{Cr}$ $L_{2,3}$ edges. The changing line shape of the XMCD spectra allows a Monte Carlo based analysis of the jump rates which are compared to theoretically calculated rates. The jump rates measured using XMCD are many orders of magnitude higher than those predicted by a thermal diffusion model, but close to those estimated using a quantum tunneling model.

The $\mathrm{Au}(111)$ surface was cleaned in situ by $\mathrm{Ar}^{+}$ion sputtering and annealing cycles at $1000 \mathrm{~K}$ in a ultra high vacuum chamber connected to a $7 \mathrm{~T}$ superconducting magnet chamber with cryopumping. After such a cleaning procedure the gold single crystal shows atomically flat terraces over several hundred nanometers, separated by 
monoatomic steps, as checked by scanning tunneling microscopy. Chromium was evaporated by electron beam heating of a Mo crucible containing high purity $\mathrm{Cr}$ crystallites. The evaporations were performed for various substrate temperatures ranging from $10 \mathrm{~K}$ to $300 \mathrm{~K}$ in the cryopumped magnet chamber under a pressure below $1 \times$ $10^{-8} \mathrm{~Pa}$. The deposited amount of $\mathrm{Cr}$ was estimated with the XAS edge jump height of the $\mathrm{Cr} L_{2,3}$ edges as discussed in Ref. [16]. The XMCD experiments were performed on beam line ID08 at the European Synchrotron Radiation Facility (ESRF). The x-ray absorption spectra at the $\mathrm{Cr} L_{2,3}$ edges were recorded for left and right circularly polarized light with a $7 \mathrm{~T}$ applied magnetic field, parallel or antiparallel to the light polarization vector. The XMCD spectra were recorded with the incoming light normal to the surface. A set of XMCD spectra for the two light helicities is measured in about 5 min. During the whole depositionmeasure procedure the sample is mounted on a cryogenic sample holder with a cold reservoir at $4 \mathrm{~K}$ located in the gap center of the liquid helium surrounded superconducting magnet.

As a function of $\mathrm{Cr}$ coverage and recording time after the deposition, different shapes of the $\mathrm{Cr} L_{2,3}$ XMCD spectra are observed (Fig. 1). For a surface density of $\sim 0.05$ atom $/ \mathrm{nm}^{2}$ (0.004 atomic layers), random deposition simulations show that $99 \%$ of the adatoms are isolated

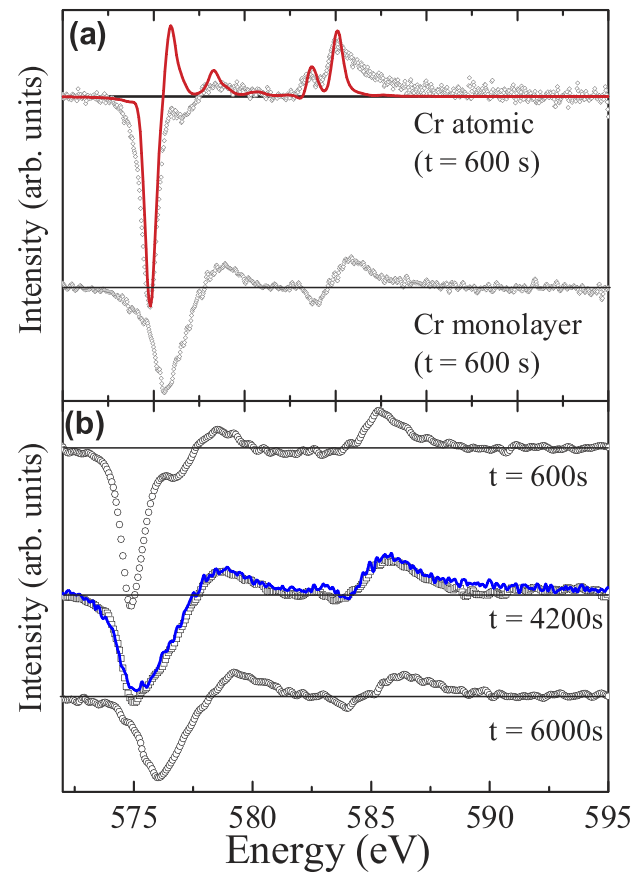

FIG. 1 (color online). (a) XMCD spectra for a collection of isolated atoms $\left(\approx 0.05\right.$ atom $\left./ \mathrm{nm}^{2}\right)$ and for a full monolayer $\mathrm{Cr}$ film $\left(\approx 14\right.$ atoms $\left./ \mathrm{nm}^{2}\right)$, deposited at $10 \mathrm{~K}$. The continuous line is a multiplet-based calculation for a single $\mathrm{Cr}$ atom with a $d^{5}$ electronic configuration. (b) Time evolution of the XMCD for a surface adatom density of $\approx 0.7$ atom $/ \mathrm{nm}^{2}$ on $\mathrm{Au}(111)$ deposited at $10 \mathrm{~K}$. The line is a linear combination of the experimental atomic and bulklike XMCD spectra. after deposition. The XMCD spectrum with characteristic multiplet features for a very low $\mathrm{Cr}$ surface density of $\sim 0.05$ atom $/ \mathrm{nm}^{2}$ differs notably from the spectrum obtained for a full $\mathrm{Cr}$ atomic layer $\left(14\right.$ atoms $\left./ \mathrm{nm}^{2}\right)$ [Fig. 1(a)]. The XMCD spectrum obtained for the monolayer situation is characteristic of highly coordinated $\mathrm{Cr}$ atoms and arises from the weak antiferromagnetic susceptibility of $\mathrm{Cr}$. The atomiclike spectrum is in excellent agreement with atomic multiplet calculations [17] for isolated $\mathrm{Cr}$ atoms with a $d^{5}$ electronic ground state configuration [Fig. 1(a)].

The isolated character of the $\mathrm{Cr}$ adatoms is also confirmed by the analysis of XMCD magnetization curves shown in Fig. 2, obtained by recording the maximum XMCD intensity as a function of the applied magnetic field. The vertical scale is rescaled in Bohr magnetons per atom by applying sum rules [18] to the complete XMCD spectrum, with the number of $d$ holes being 5.43, obtained from electronic structure calculations. The magnetization curve is well described by a Brillouin function representing the paramagnetic phase of a collection of isolated noninteracting adatoms. The total magnetic moment extracted from a Brillouin function fit is $m=4.5 \pm$ $0.4 \mu_{B}$ per atom, in good agreement with fully relativistic electronic structure calculations using the embedding technique within the Korringa-Kohn-Rostoker method [19] giving $m_{s}=4.45 \mu_{B}$ and $m_{l}=-0.02 \mu_{B}$ for a single $\mathrm{Cr}$ atom in an fcc site on $\mathrm{Au}(111)$. In the low $\mathrm{Cr}$ coverage limit, the initial XMCD spectrum progressively loses its atomic character as a function of time due to aggregation of adatoms through surface diffusion. Figure 1(b) displays the changes in the XMCD measured for a surface density of $\sim 0.7$ atom $/ \mathrm{nm}^{2}$. Monte Carlo simulations show that for a Cr density of $\sim 0.7$ atoms $/ \mathrm{nm}^{2}$ [Fig. 1(b)] a collection of isolated adatoms evolves towards dimers and trimers which can be considered as stable [20,21]. The resulting $\mathrm{XMCD}$ spectrum has a shape identical to that recorded for a $\mathrm{Cr}$ monolayer. This shows that, within the experimental

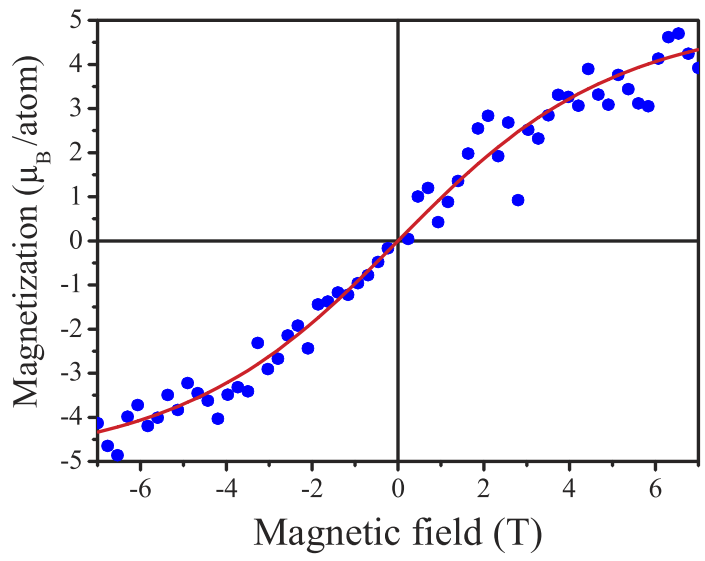

FIG. 2 (color online). XMCD magnetization curve recorded at the $L_{3} \mathrm{Cr}$ edge at $T=10 \mathrm{~K}$ for a collection of isolated $\mathrm{Cr}$ atoms. The full line is a Brillouin function fit. 
resolution, two types of XMCD spectra can be distinguished, an atomiclike spectrum for isolated $\mathrm{Cr}$ atoms and a bulklike spectrum as soon as the $\mathrm{Cr}$ atoms aggregate to form dimers.

Alternative origins of the time evolution can be ruled out. A collection of isolated adatoms unexposed to the beam shows the same changes in the XMCD spectrum for a delayed first measurement implying that the $\mathrm{x}$-ray beam is indeed nonperturbing. Surface contamination can definitely be excluded since: (i) The time-evolved XMCD spectra for single adatoms is identical to the spectrum for a clean $\mathrm{Cr}$ monolayer; (ii) Increasing the substrate temperature from $10 \mathrm{~K}$ to $40 \mathrm{~K}$ significantly speeds up the time evolution of the XMCD spectra; (iii) No evolution is observed at low temperature for small clusters containing several tens of atoms deposited at higher temperatures. (iv) Exposure to several langmuirs of $\mathrm{CO}$ leads to spectra with pronounced multiplet structures, different from the time-evolved spectra.

An XMCD spectrum at a given time during diffusion can therefore be decomposed as a linear combination of atomic and bulk spectra [Fig. 1(b) middle spectrum, solid line]. This decomposition allows the ratio, $R$, of isolated $\mathrm{Cr}$ adatoms to the total number of $\mathrm{Cr}$ adatoms to be determined during the diffusion process. This is illustrated in the inset of Fig. 3 for a $\sim 0.05$ atom $/ \mathrm{nm}^{2}$ surface atomic density of $\mathrm{Cr}$. The approach also allows $R$ to be determined for different initial surface coverages. Figure 3 displays the coverage dependence of $R$ calculated from the deconvolution of the XMCD spectra recorded during the first $600 \mathrm{~s}$ after the end of the deposition. The proportion $R$ obtained by a random walk simulation is also shown in Fig. 3 (lines) for two different numbers of Monte Carlo steps $\left(N_{\mathrm{MC}}\right)$. The as deposited Monte Carlo situation $\left(N_{\mathrm{MC}}=0\right)$ does not fit the experimental data, and 35 Monte Carlo steps are necessary to reproduce the experimental data points between 10 and $30 \mathrm{~K}$. These 35 Monte Carlo steps correspond to the evolution of the system during the $600 \mathrm{~s}$ it takes to reliably record the first XMCD spectra. At $40 \mathrm{~K}$, about 3500 Monte Carlo steps are needed. Above $40 \mathrm{~K}$, the time evolution is too fast to be detected experimentally. In the temperature range from 10 to $30 \mathrm{~K}$, the number of Monte Carlo steps necessary to reproduce the time evolution of the system after $600 \mathrm{~s}$ is temperature independent, and therefore rules out any thermal activated effects. From the time evolution of $R$, via a scaling to the Monte Carlo number of steps, one deduces an average experimental jump rate $\left(\Gamma_{\exp }\right)$ of the $\mathrm{Cr}$ adatoms which is shown in Table I. Also shown in Table $I$ is the surface diffusion coefficient $\left(D_{\text {exp }}\right)$ which is given by $D_{\exp }=\frac{\sqrt{3}}{4} r_{0}^{2} \Gamma_{\text {exp }}$ for an fcc(111) surface, where $r_{0}$ is the lattice parameter which is $2.86 \AA$ for $\mathrm{Au}(111)$. The time dependence of $R$ shows two different diffusion regimes for the $\mathrm{Cr}$ adatoms, a temperature independent one below $40 \mathrm{~K}$ and a temperature dependent one above.

In the thermally activated regime, at $40 \mathrm{~K}$, one can deduce the energy barrier height, $E_{a}$, through the Arrhenius

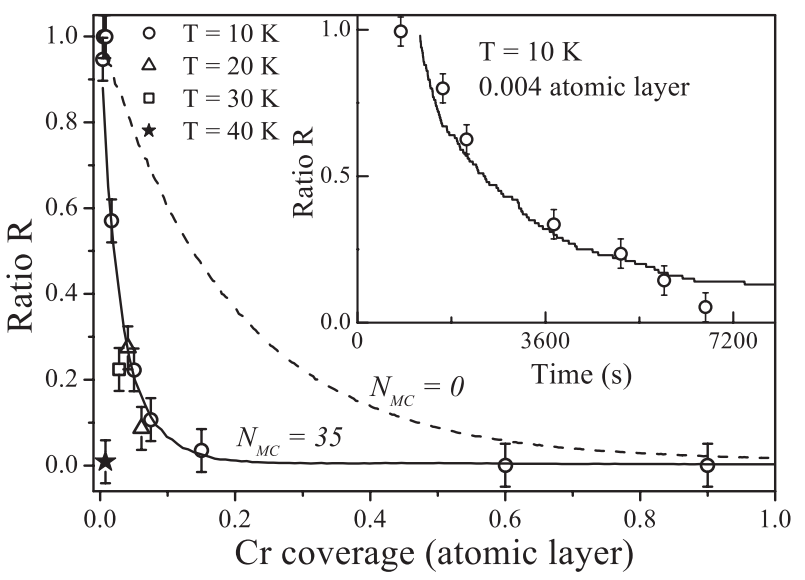

FIG. 3. Ratio $R$ of the number of isolated $\mathrm{Cr}$ adatoms to the total number of deposited $\mathrm{Cr}$ atoms as a function of coverage, as determined from XMCD spectra for several coverages and temperatures. Lines represent Monte Carlo simulation of $R$ for a random atomic deposition, $N_{\mathrm{MC}}=0$ (dashed line) and after $N_{\mathrm{MC}}=35$ Monte Carlo steps (solid line) for a random atomic diffusion. The error on the coverage is less than $10 \%$. Inset: time evolution of $R$ at $10 \mathrm{~K}$ for a 0.004 atomic layer $\mathrm{Cr}$ deposit $\left(\sim 0.05\right.$ atom $\left./ \mathrm{nm}^{2}\right)$ (disks) and corresponding Monte Carlo simulation (solid line).

formula $[22,23]$

$$
\Gamma_{\text {therm }}=\Gamma_{0} \exp \left(-\frac{E_{a}}{k_{B} T}\right)
$$

where $k_{B}$ is the Boltzman constant, $T$ the temperature, $\Gamma_{0}$ is a prefactor which contains dynamical quantities and $\Gamma_{\text {therm }}$ is the thermal jump rate. Typically $\Gamma_{0} \simeq 10^{12}-10^{13} \mathrm{~Hz}$ for most surfaces [24]. The value of $E_{a}$ is found to be $\simeq 100 \pm$ $15 \mathrm{meV}$, in excellent agreement with a theroretical value of $98 \mathrm{meV}$ obtained via the nudged elastic band method in the framework of the modified embedded atom method [14,25-27]. The parameters given in Refs. [26,27] for the gold and chromium potentials, respectively, have been used while the cross-potential between $\mathrm{Au}$ and $\mathrm{Cr}$ has been fitted with first-principles calculations. The value of $E_{a}$ obtained is similar to those obtained on related systems [15].

The temperature independence of the experimental jump rate between 10 and $30 \mathrm{~K}$ is shown in Table I. These values show a striking contrast to the temperature dependence of a thermally activated jump rate. The temperature independent behavior of the site-to-site hopping rates below $40 \mathrm{~K}$ is a hallmark of a quantum tunneling process [7-9] implying that classical thermally activated surface diffusion can be excluded as the origin of the $\mathrm{Cr}$ adatom diffusion in this temperature regime. Indeed, in the considered temperature range, the de Broglie wavelength of a $\mathrm{Cr}$ adatom $\lambda_{B}=$ $2 \pi \hbar / \sqrt{3 m k_{B} T}$ becomes comparable to the site separation distance $(\approx 1 \AA)$, making quantum tunneling possible ( $\hbar$ is the reduced Planck constant and $m$ the mass of the $\mathrm{Cr}$ atom). A full quantum treatment of the surface diffusion, taking into account the surface potential and its dynamical aspects [7], cannot be presented here. However, a useful 
TABLE I. Experimental jump rates, $\Gamma_{\text {exp }}$, the corresponding diffusion coefficients, $D_{\text {exp }}$, and thermally activated diffusion jump rates, $\Gamma_{\text {therm }}$, calculated for an energy activation barrier $E_{a}=100 \mathrm{meV}$. The prefactor $\Gamma_{0}$ is $5 \times 10^{12} \mathrm{~Hz}$. The tunneling jump rate, $\Gamma_{\text {tun }}$, has been calculated for a one-dimensional parabolic double-well potential.

\begin{tabular}{cccc}
\hline \hline$T(\mathrm{~K})$ & $\Gamma_{\text {exp }}(\mathrm{Hz})$ & $D_{\text {exp }}\left(\mathrm{cm}^{2} \mathrm{~s}^{-1}\right)$ & $\Gamma_{\text {therm }}(\mathrm{Hz})$ \\
\hline 10 & $(3.7 \pm 0.9) \times 10^{-2}$ & $(1.3 \pm 0.3) \times 10^{-17}$ & $1.3 \times 10^{-37}$ \\
20 & $(4.2 \pm 0.9) \times 10^{-2}$ & $(1.5 \pm 0.3) \times 10^{-17}$ & $8.0 \times 10^{-13}$ \\
30 & $(4.2 \pm 0.6) \times 10^{-2}$ & $(1.5 \pm 0.2) \times 10^{-17}$ & $1.5 \times 10^{-4}$ \\
40 & $(2.0 \pm 0.3)$ & $(7.0 \pm 1.0) \times 10^{-16}$ & 2.0 \\
\hline \hline
\end{tabular}

analytical formula for the tunneling jump rate can be derived for a one-dimensional parabolic double-well potential. For the fundamental energy level, the tunneling jump rate, $\Gamma_{\text {tun }}$, is approximated by [28]

$$
\Gamma_{\text {tun }}=\frac{2 \omega}{\pi^{3 / 2}} \sqrt{\frac{2 E_{a}}{\hbar \omega}} \exp \left(-\frac{2 E_{a}}{\hbar \omega}\right)
$$

with $\omega=\sqrt{2 E_{a} / m \cdot b^{2}} ; b$ is the barrier width $[b=0.8 \AA$ for $\mathrm{Au}(111)]$. With $E_{a}=100 \pm 15 \mathrm{meV}$, one obtains $\Gamma_{\text {tun }} \approx 10^{-6}-10^{-2} \mathrm{~Hz}$. Although this model represents a crude simplification, one can notice that the tunneling siteto-site hopping rate is more than 30 orders of magnitude larger than the thermal rate at $10 \mathrm{~K}$, in far better agreement with the experimental values.

In summary, the sensitivity of XMCD to the surface atomic coordination has been used to study lowtemperature diffusion of $\mathrm{Cr}$ adatoms on $\mathrm{Au}(111)$. The analysis of the time evolution of the XMCD spectra via Monte Carlo simulations has allowed the determination of the jump rates. In the thermally activated regime, (i.e., $T>$ $40 \mathrm{~K}$ ), the measured energy diffusion barrier is $\sim 100 \mathrm{meV}$ for $\mathrm{Cr}$ adatoms migrating on $\mathrm{Au}(111)$, in good agreement with the theoretical value. For $T<40 \mathrm{~K}$, where the classical description of thermal diffusion does not allow further adatom diffusion, the motion of $\mathrm{Cr}$ adatoms was observed over a time scale of several hundred seconds. This extremely fast diffusion of $\mathrm{Cr}$ adatoms on $\mathrm{Au}(111)$ is attributed to quantum tunneling.

We are grateful to N.B. Brookes for constant support on ID08 and to Professor M. F. Crommie, F. Bardou, C. Goyhenex, J. Repp, M. Romeo, D. Weinmann, and A. Boeglin for fruitful discussions. The work of B. L. was supported by the Center for Computational Materials Science (Contract No. GZ 45.531). The late Kenneth Larsson provided valuable insight into this work.

*Present address: Synchrotron SOLEIL, L'Orme des Merisiers, Saint Aubin, BP 48, F-91192 Gif sur Yvette, France.

Electronic address: philippe.ohresser@synchrotronsoleil.fr
${ }^{\dagger}$ Present address: Diamond Light Source, Chilton, Didcot, OX11 0QX, United Kingdom

[1] G. Binnig et al., Phys. Rev. Lett. 49, 57 (1982).

[2] H.C. Manoharan, C.P. Lutz, D. M. Eigler, Nature (London) 403, 512 (2000).

[3] A. J. Heinrich et al., Science 298, 1381 (2002).

[4] L. de Broglie, Nature (London) 112, 540 (1923).

[5] L. J. Lauhon and W. Ho, Phys. Rev. Lett. 85, 4566 (2000).

[6] A. Yazdani, Nature (London) 409, 471 (2001), and refs. therein.

[7] V. Pouthier and J. C. Light, J. Chem. Phys. 113, 1204 (2000).

[8] L. Y. Chen and S.C. Ying, Phys. Rev. Lett. 73, 700 (1994).

[9] T. R. Mattson, U. Engberg, and G. Wahnström, Phys. Rev. Lett. 71, 2615 (1993).

[10] P. Gambardella et al., Phys. Rev. Lett. 88, 047202 (2002).

[11] P. Gambardella et al., Science 300, 1130 (2003).

[12] J. Stöhr, J. Electron Spectrosc. Relat. Phenom. 75, 253 (1995).

[13] J. Repp et al., Phys. Rev. Lett. 91, 206102 (2003).

[14] H. Bulou and B. Lazarovits (unpublished).

[15] S. Rohart et al., Surf. Sci. 559, 47 (2004).

[16] P. Ohresser et al., Phys. Rev. B 62, 5803 (2000).

[17] See, e.g., F. de Groot, Chem. Rev. 101, 1779 (2001).

[18] B. T. Thole et al., Phys. Rev. Lett. 68, 1943 (1992); P. Carra et al., Phys. Rev. Lett. 70, 694 (1993).

[19] See, e.g., P. Weinberger, Electron Scattering Theory for Ordered and Disordered Matter (Clarendon, Oxford, 1990).

[20] J. A. Venables, G. D. T. Spiller, and M. Handbücken, Rep. Prog. Phys. 47, 399 (1984).

[21] T. Jamneala, V. Madhavan, and M. F. Crommie, Phys. Rev. Lett. 87, 256804 (2001).

[22] S. Arrhenius, Z. Phys. Chem. (Leipzig) 4, 226 (1889).

[23] T. Ala-Nissila, R. Ferrando, S. C. Ying, Adv. Phys. 51, 949 (2002).

[24] S. Ovesson et al., Phys. Rev. B 64, 125423 (2001).

[25] H. Jónsson, G. Mills, and K. W. Jacobsen, in Classical and Quantum Dynamics in Condensed Phase Simulations, edited by B.J. Berne, G. Ciccotti, and D.F. Coker (World Scientific, Singapore, 1998), p. 385.

[26] Byeong-Joo Lee et al., Phys. Rev. B 64, 184102 (2001).

[27] Byeong-Joo Lee, Jae-Hyeok Shim, and M. I. Baskes, Phys. Rev. B 68, 144112 (2003).

[28] E. Merzbacher, Quantum Mechanics (J. Wiley, New York, 1970). 